\title{
Unanticipated Region- and Cell-Specific Downregulation of Individual KChIP Auxiliary Subunit Isotypes in Kv4.2 Knock-Out Mouse Brain
}

\author{
Milena Menegola and James S. Trimmer \\ Department of Pharmacology, School of Medicine, University of California, Davis, California 95616
}

\begin{abstract}
Kv4 family voltage-gated potassium channel $\alpha$ subunits and Kv channel-interacting protein (KChIP) and dipeptidyl aminopeptidase-like protein subunits comprise somatodendritic A-type channels in mammalian neurons. Recently, a mouse was generated with a targeted deletion of Kv4.2, a Kv4 $\alpha$ subunit expressed in many but not all mammalian brain neurons. $K v 4.2^{-/-}$mice are grossly indistinguishable from wild-type (WT) littermates. Here we used immunohistochemistry to analyze expression of component Kv4 and KChIP subunits of A-type channels in WT and $K v 4.2^{-/-}$brains. We found that the expression level, and cellular and subcellular distribution of the other prominent brain Kv4 family member Kv4.3, was indistinguishable between WT and $K v 4.2^{-/-}$samples. However, we found unanticipated regional and cell-specific decreases in expression of KChIPs. The degree of altered expression of individual KChIP isoforms in different regions and neurons precisely follows the level of Kv4.2 normally found at those sites and presumably their extent of association of these KChIPs with Kv4.2. The dramatic effects of Kv4.2 deletion on KChIP expression suggest that, in addition to previously characterized effects of KChIPs on the functional properties, trafficking, and turnover rate of Kv4 channels, Kv4:KChIP association may confer reciprocal Kv4.2-dependent effects on KChIPs. The impact of Kv4.2 deletion on KChIP expression also supports the major role of KChIPs as auxiliary subunits of $\mathrm{Kv} 4$ channels.
\end{abstract}

Key words: cerebellum; dendrite; excitability; hippocampus; immunohistochemistry; ion channel

\section{Introduction}

Somatodendritic A-type voltage-gated potassium channels comprising pore-forming and voltage-sensing Kv $4 \alpha$ subunits ( $\mathrm{Kv} 4 \mathrm{~s}$ ), and $\mathrm{Kv}$ channel-interacting protein (KChIP) and/or dipeptidyl aminopeptidase-like protein (DPPX) auxiliary subunits, regulate excitability of neuronal somata and dendrites (Jerng et al., 2004b). The predominant mammalian brain Kv4s are Kv4.2 and Kv4.3, found in association with KChIP1-KChIP4 (Serodio and Rudy, 1998; Rhodes et al., 2004). Immunohistochemical staining using Kv4- and KChIP-specific monoclonal antibodies revealed that different KChIPs are concentrated in neuronal somata and dendrites in which their cellular and subcellular distribution overlaps, in an isoform-specific manner, with that of Kv4.2 and Kv4.3 (Rhodes et al., 2004; Strassle et al., 2005). For example, immunoreactivity for KChIP2 and Kv4.2 is concentrated in dendrites of hippocampal and neocortical pyramidal cells, for KChIP2, KChIP3, Kv4.2, and Kv4.3 in dendrites of hippocampal dentate granule cells, and for KChIP1 and Kv4.3 in somata and

\footnotetext{
Received June 29, 2006; revised 0ct. 11, 2006; accepted 0ct. 13, 2006.

This work was supported by National Institutes of Health Grants NS42225 and NS34383 (J.S.T.). M.M. acknowledges a postdoctoral fellowship from the Epilepsy Foundation through generous support of the American Epilepsy Society and the Milken Family Foundation. We thank Andrew Siegel for technical support and Drs. Kenneth J. Rhodes and Hiroaki Misonou for helpful advice and critical readings of this manuscript.

Correspondence should be addressed to Dr. James S. Trimmer, Department of Pharmacology, School of Medicine, 3502 Genomic and Biomedical Sciences Facility, University of California, One Shields Avenue, Davis, CA 95616 E-mail: jtrimmer@ucdavis.edu.

DOI:10.1523/JNEUROSCI.2783-06.2006

Copyright $\odot 2006$ Society for Neuroscience $\quad$ 0270-6474/06/2612137-06\$15.00/0
}

dendrites of hippocampal, striatal, and neocortical interneurons. The precise pairings of specific KChIPs and Kv4s with one another suggests specific requirements for Kv4:KChIP coassembly and a prominent role for KChIPs as component subunits of Kv4 channels.

Recently, a mouse with a targeted deletion of $\mathrm{Kv} 4.2$ $\left(K v 4.2^{-\prime-}\right)$ was generated (Guo et al., 2005; Hu et al., 2006). Given the widespread, high-level expression of Kv4.2 in central neurons, it was surprising that $K v 4.2^{-/-}$mice are grossly normal in appearance, behavior, and body weight (Hu et al., 2006). Given the partially overlapping expression of the functionally similar $\mathrm{Kv} 4.3$ in brain neurons, one possibility is that $\mathrm{Kv} 4.3$ upregulation in $K v 4.2^{-/-}$brain confers functional compensation. The genetic deletion of a prominent candidate binding partner allows for analyses of the subsequent impact on KChIPs. Here we used immunohistochemistry using subunit-specific monoclonal antibodies to analyze expression of component $\mathrm{Kv} 4$ and KChIP subunits of somatodendritic A-type channels in wild-type (WT) and Kv4.2. $2^{-/-}$brains.

\section{Materials and Methods}

Antibody production. We previously described all monoclonal antibodies used here (Bekele-Arcuri et al., 1996; Rhodes et al., 2004; Misonou et al., 2006); all are available from NeuroMab (www.neuromab.org), a not-forprofit supplier of monoclonal antibodies administered through the University of California. We also previously described rabbit polyclonal antiKv2.1 antibody KC (Trimmer, 1991). 
Tissue preparation. We performed all animal procedures in strict accordance with the National Institutes of Health Guide for the Care and Use of Laboratory Animals. We obtained adult male $K v 4.2^{-1-}$ mice in the FVB background (Guo et al., 2005; Hu et al., 2006) from Dr. Thomas L. Schwarz (Harvard University, Boston, MA) and Dr. Jeanne Nerbonne (Washington University School of Medicine, St. Louis, MO). We obtained age and sex-matched WT (FVB) animals from Taconic (Germantown, NY). We anesthetized animals intraperitoneally with $60 \mathrm{mg} / \mathrm{kg}$ pentobarbital and perfused with $20 \mathrm{ml}$ of ice-cold saline solution, followed by a slow perfusion of $80 \mathrm{ml}$ of $4 \%$ paraformaldehyde in phosphate-buffered solution. We immediately removed brains from the skull and cryoprotected and cut sagittal brain sections ( $40 \mu \mathrm{m}$ thick) using a freezing stage sliding microtome.

Multiple-label immunofluorescence staining of brain sections. We blocked free-floating sections with $10 \%$ goat serum in phosphate buffer containing $0.3 \%$ Triton X-100 (vehicle) and then incubated them overnight at $4^{\circ} \mathrm{C}$ in vehicle containing different combinations of antibodies (either rabbit polyclonal-mouse monoclonal or mouse monoclonal antibodies of different IgG isotypes). We then incubated sections in speciesor isotype-specific Alexa-conjugated secondary antibodies (Invitrogen, Carlsbad, CA) as described previously (Strassle et al., 2005).

Imaging/data analysis. We recorded images with a digital CCD camera installed on a Axioskop-2 Zeiss (Oberkochen, Germany) microscope with a Zeiss Fluar $10 \times / 0.50$ numerical aperture lens using Axiovision software and manually tiled them using Photoshop (Adobe Systems, San Jose, CA). We performed fluorescence intensity measurements in hippocampus using NIH ImageJ software by selecting a $60 \times 60 \mu \mathrm{m}$ area from the molecular layer of dentate gyrus and from stratum radiatum of CA1. We then subtracted the fluorescence intensity measured on a comparable region of a section from the same animal but incubated with secondary antibody alone to determine fluorescence staining intensity contributed by primary antibody. We used similar methods in cerebellum (anterior and posterior) and striatum, except that the area selected for intensity measurements corresponded to $50 \times 50$ and $400 \times 400 \mu \mathrm{m}$ squares, respectively. We obtained values from comparable sections obtained from five $\mathrm{Kv} 4.2^{-/-}$and five age-, strain-, and sex-matched WT.

\section{Results}

To address effects of genetic ablation of Kv4.2 on the distribution of other subunits involved in comprising somatodendritic A-type channels, we used multiple-labeling immunofluorescence staining to determine their localization and expression level in WT and $K v 4.2^{-1-}$ brain sections. We focused our analyses on hippocampus, cerebellum, and striatum, brain regions in which somatodendritic A-channel subunit distribution and function has been most intensely studied (for review, see Jerng et al., 2004b).

\section{Hippocampus}

In general, the staining we observed for component Kv4s and KChIPs in WT mouse hippocampus strongly resembled that in rat (Rhodes et al., 2004). In WT sections, Kv4.2 was robustly detected in strata oriens and radiatum of CA1-CA3 corresponding to apical and basal dendrites of pyramidal cells and in the molecular layer of dentate gyrus in which dendrites of dentate granule cells are localized (Fig. $1 A$, left). Kv4.3 was present and colocalized with Kv4.2 in dendrites of principal cells of CA3 and dentate gyrus (Fig. $1 B$, left). Unlike Kv4.2, Kv4.3 was not expressed in pyramidal neurons of CA1 (Fig. $1 B$, left). Kv4.3 was also prominently detected in somata and dendrites of interneurons throughout hippocampus (Fig. $1 B$, left); these cells lack appreciable Kv4.2 (Fig. $1 A$, left).

KChIP1 was present and coexpressed with Kv4.3 on somata and dendrites of interneurons scattered throughout the hippocampus (Fig. $1 C$, left). KChIP2 showed a very similar cellular and subcellular distribution to $\mathrm{Kv} 4.2$, with strong staining on dendrites of dentate granule cells and CA3-CA1 pyramidal cells

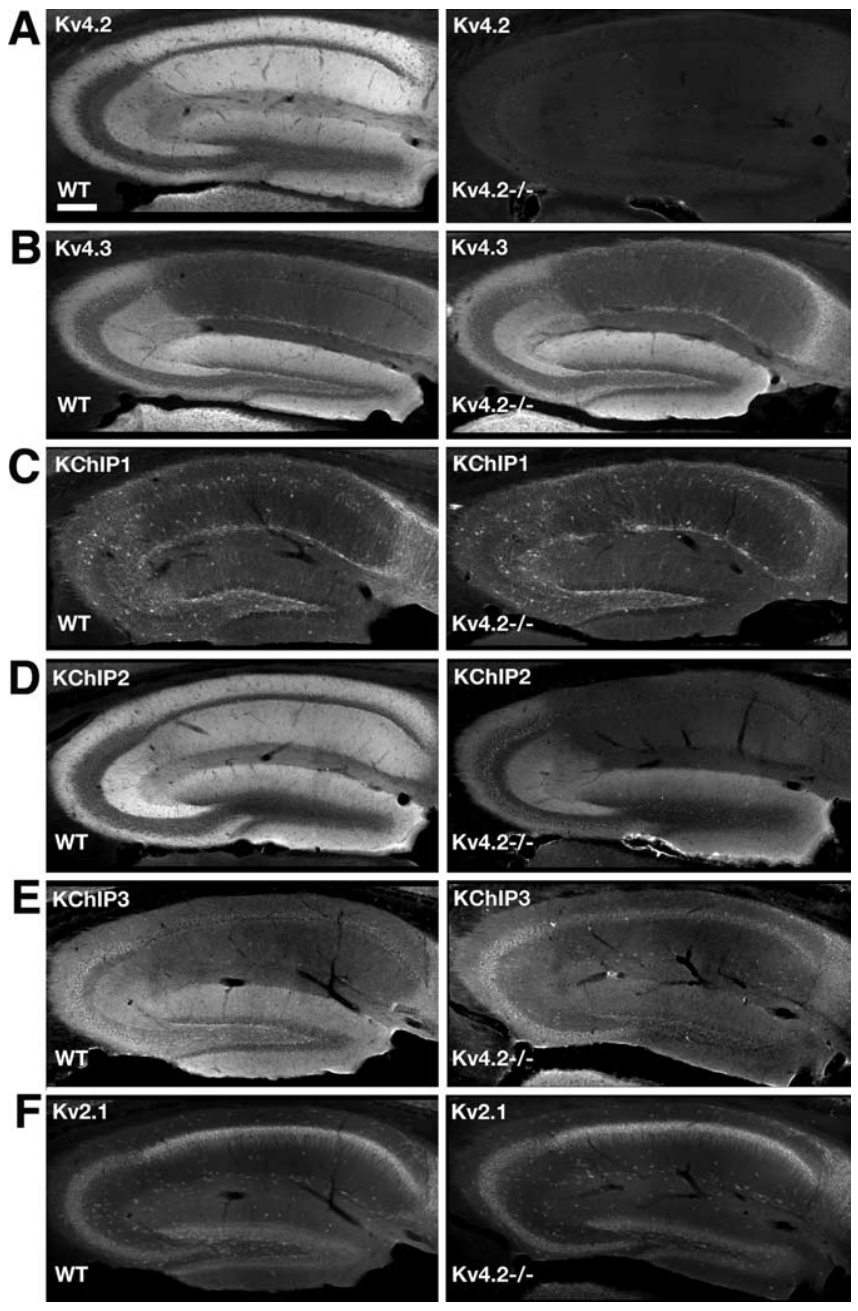

Figure 1. Expression of Kv4 and KChIP subunits in WT and Kv4.2 $2^{-/-}$hippocampi. WT and Kv4.2 $2^{-/-}$brain sections immunostained for potassium channel subunits as indicated. Scale bar, $200 \mu \mathrm{m}$.

(Fig. $1 D$, left). KChIP3 was most prevalent in the molecular layer of dentate gyrus (Fig. $1 E$, left), in a pattern that was similar to Kv4.2, KChIP2, and Kv4.3. We observed little KChIP3 in other regions of hippocampus.

We observed a number of changes in comparable $K v 4.2^{-/-}$ brain sections. As expected, Kv4.2 immunoreactivity was eliminated in $K v 4.2^{-/-}$sections (Fig. $1 \mathrm{~A}$, right). Interestingly, there were no apparent changes in the cellular or subcellular expression of Kv4.3 (Fig. $1 B$, right). For example, in CA1, we observed that $\mathrm{Kv} 4.3$ remains restricted to interneurons and was not on apical or basal dendrites of CA1 pyramidal neurons (Fig. $1 \mathrm{~B}$, right). Moreover, there were no apparent changes in Kv4.3 expression in $K v 4.2^{-/-}$hippocampus in those areas in which Kv4.2 and Kv4.3 are normally coexpressed and colocalized, such as dentate granule and CA3 pyramidal cell dendrites (Fig. $1 B$, right). Consistent with these observations, we saw no significant compensatory increases in Kv4.3 expression level in quantitative measurements of Kv4.3 fluorescence in the dentate gyrus molecular layer or in stratum radiatum of CA1 (Fig. $2 A, B$ ), in which staining emanates from interneurons.

In contrast, we found that KChIP2 and KChIP3 exhibited 

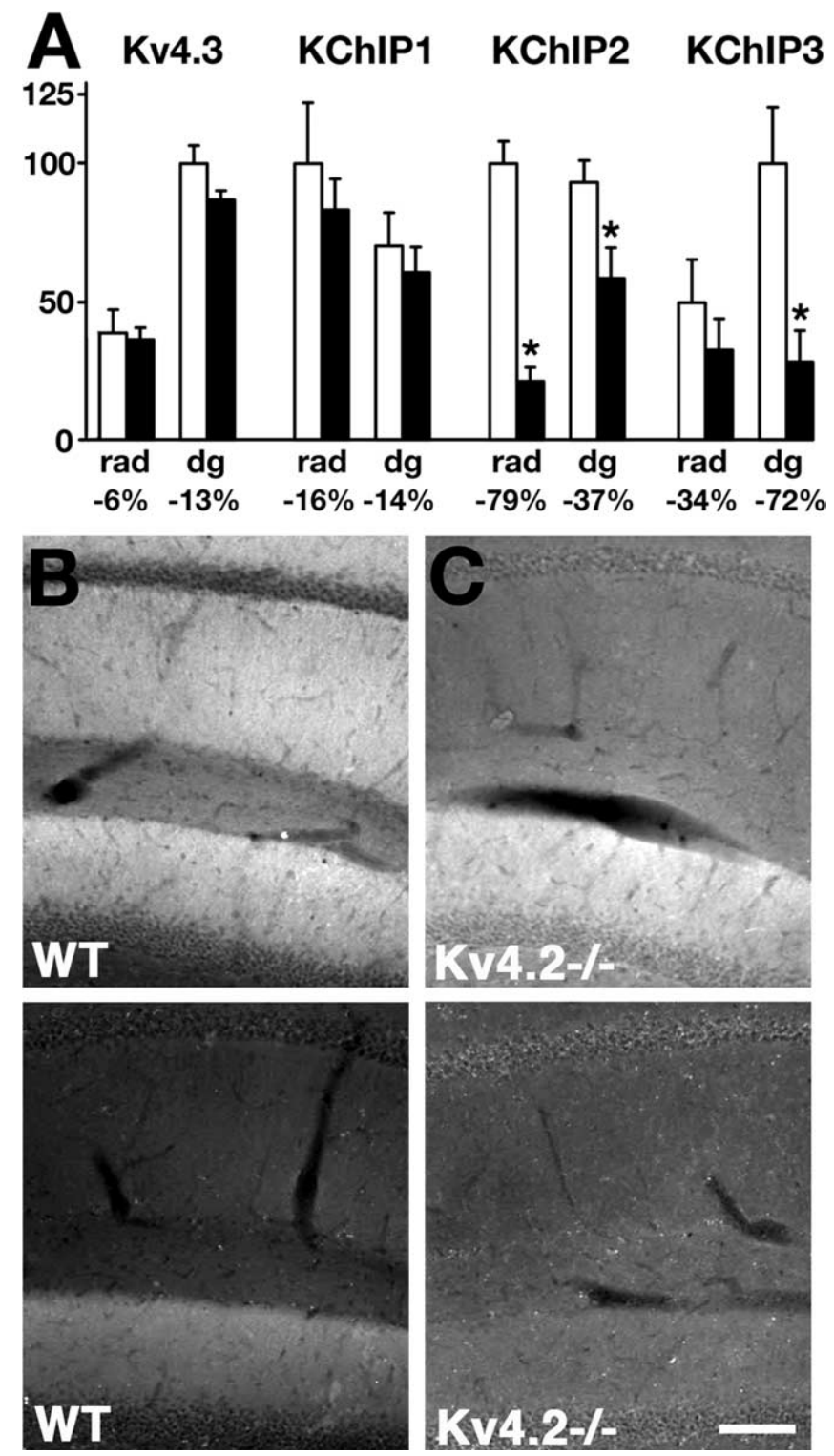

Figure 2. Quantitative analysis of Kv4 and KChIP subunits in WT and Kv4.2 $2^{-/-}$hippocampi. $A$, Fluorescence intensity measurements from a $60 \times 60 \mu \mathrm{m}$ area in stratum radiatum (rad) of CA1 adjacent to the pyramidal cell layer and in the molecular layer of dentate gyrus ( $\mathrm{dg}$ ) adjacent to the hippocampal fissure in five WT (white bars) and five $K v 4.2^{-/-}$(black bars) mice. We set the highest mean value for a given subunit at $100 \%$ and subsequently normalized the remaining three mean values. Values are mean $\pm S E$. Asterisks mark values obtained from Kv4. $2^{-1-}$ sections that are significantly different $(p<0.05 ; n=5)$ from WT values. $\boldsymbol{B}, \boldsymbol{C}$, Higher-magnification views of WT and $K v 4.2^{-/-}$brain areas used for quantification of fluorescence intensity for KChIP2 (B) and KChIP3 (C). Scale bar, $100 \mu \mathrm{m}$.

pronounced subfield-specific changes upon Kv4.2 ablation. KChIP2 in Kv4.2 $2^{-/-}$hippocampus was dramatically $(>75 \%)$ (Fig. 2A) decreased in strata oriens and radiatum of CA1 (Fig. $1 D$, right), regions that, in WT, exhibit strong KChIP2 labeling that colocalizes with Kv4.2 (Fig. $1 A, D$, left). KChIP2 was also decreased in strata radiatum and oriens of CA 3 and in the molecular layer of dentate gyrus, although some KChIP2 was still clearly present. The decrease of KChIP2 in the molecular layer of dentate gyrus was less dramatic than we observed in CA1 but was still significant (Fig. 2A,B). A comparison of the top left and right panels of Figure $2 C$ reveals the preferential loss of KChIP2 in CA1.

KChIP3 in the molecular layer of dentate gyrus (Fig. 1E, left) is dramatically ( $\sim 80 \%$ ) (Fig. $2 \mathrm{~A}$ ) reduced in $\mathrm{Kv} 4.2^{-/-}$sections (Fig. $1 E$, right). The low level of KChIP3 in stratum radiatum of $\mathrm{CA} 1$ is also depressed in $\mathrm{Kv} 4.2^{-1-}$ hippocampus (Figs. $1 \mathrm{E}$, right, $2 A)$. Overall, the effects of Kv4.2 ablation on KChIP3 are more robust in dentate gyrus than in other hippocampal subfields. This is especially apparent in the higher-magnification view of KChIP3 staining (Fig. $2 C$, bottom) and in the measured ratio of staining in CA1 versus dentate gyrus in Figure $2 B$, in which we observed a pattern of overall response to loss of $\mathrm{Kv} 4.2$ that is complementary to that observed for KChIP2. We saw little change in expression and localization of the interneuronrestricted KChIP1 in WT versus $\mathrm{Kv} 4.2^{-/-}$hippocampus (Figs. $1 C, 2 A$ ). We did observe a decrease in KChIP1 in subiculum (Fig. $2 A)$. Kv2.1, which constitutes a major somatodendritic delayed rectifier Kv channel in central neurons (Trimmer, 1991), is also unchanged in WT versus $\mathrm{Kv} 4.2^{-/-}$hippocampus (Fig. $1 F$ ). Because Kv1.4, a component $\alpha$ subunit of a distinct class of neuronal A-type channels, is ectopically upregulated in hearts of $K v 4.2^{-\prime-}$ mice (Guo et al., 2005), we also examined Kv1.4 staining in WT and $K v 4.2^{-/-}$hippocampus; however, we observed no obvious changes in overall Kv1.4 expression or localization (supplemental Fig. $1 A$, available at www.jneurosci.org as supplemental material).

\section{Cerebellum}

Expression of Kv4s and KChIPs in WT cerebellum was also similar to that in rat (Rhodes et al., 2004; Strassle et al., 2005). Expression of Kv4.2, KChIP1, and KChIP3 was evaluated relative to other potassium channels prominently expressed in cerebellum [Slo1 (Misonou et al., 2006) and Kv1.2 (Rhodes et al., 1997)]. Kv4.2 (red) was most prominent in the granule cell layer (Fig. 3A, top). We also observed KChIP1 (red) in the granule cell layer of WT cerebella (Fig. 3B, top), with an anterior $>$ posterior gradient as in rat (Strassle et al., 2005), with additional KChIP1 in the molecular layer. KChIP3 was also prominent in the WT cerebellar granule cell layer, with no obvious anterior/posterior gradient (Fig. 3C, top).

In $K v 4.2^{-/-}$cerebella, staining for $\mathrm{Kv} 4.2$ (red) is eliminated (Fig. $3 A$, bottom). We observed a large $(-83.4 \pm 5.6 \%)$ and significant ( $p=0.0006$ vs WT; $n=5$ ) decrease in KChIP1 in the $K v 4.2^{-1-}$ anterior granule cell layer (Fig. $3 B, E$ ), such that the anterior to posterior gradient of KChIP1 expression in the granule cell layer was lost (Fig. 3B, bottom). Staining for Slo1 (Fig. $3 A, B$, green) is unchanged. KChIP3 staining (red) in the granule cell layer was also greatly attenuated (Fig. $3 C, E$ ) in both anterior $(68.4 \pm 10.3 \%$ decrease; $p=0.008$ vs WT; $n=5)$ and posterior $(68.0 \pm 10.3 \%$ decrease; $p=0.006$ vs WT; $n=5) K v 4.2^{-/-}$ cerebellar cortex (Fig. 3C, bottom), whereas staining for Kv1.2 (green) in basket cell terminals and in the molecular layer is unchanged (Fig. $3 C$ ). In data not shown, prominent Kv4.3 staining was found in the WT granule cell layer in a clear posterior $>$ anterior gradient as in rat (Serodio and Rudy, 1998; Strassle et al., 2005). We saw no differences in Kv4.3, KChIP2 (data not shown), or Kv1.4 (supplemental Fig. $1 B$, available at www.jneurosci.org as supplemental material) staining in WT versus $K v 4.2^{-/-}$ cerebella.

\section{Striatum}

As in rat (Rhodes et al., 2004), striatal immunoreactivity for $\mathrm{Kv} 4.2, \mathrm{Kv} 4.3$, KChIP2, and KChIP3 is concentrated in neuropil, with additional immunoreactivity for Kv4.3 and KChIP1 in interneurons. As expected, $\mathrm{Kv} 4.2$ immunoreactivity virtually disappeared from $\mathrm{Kv} 4.2^{-/-}$striatum. Whereas Kv4.3, KChIP1, and 
KChIP3 were essentially unaffected in Kv4.2 $2^{-1-}$ striatum (Fig. 3D), KChIP2 in neuropil was significantly $(>40 \%)$ reduced (Fig. 3D,F). We observed no changes in the overall Kv1.4 expression or localization between WT and $K v 4.2^{-/-}$striata (supplemental Fig. 1C, available at www. jneurosci.org as supplemental material).

\section{Discussion}

Kv4 channels underlie somatodendritic A-type currents in neurons (Birnbaum et al., 2004; Jerng et al., 2004b). These channels are formed by four Kv $4 \alpha$ subunits in association with cytoplasmic KChIP (An et al., 2000; Rhodes et al., 2004) and/or transmembrane DPPX (Nadal et al., 2003) or dipeptidylpeptidase 10 (Jerng et al., 2004a; Zagha et al., 2005) auxiliary subunits. Coexpression of KChIP1-KChIP3, but not KChIP4, with $\mathrm{Kv} 4 \alpha$ subunits in heterologous cells dramatically increases surface expression and slows the turnover rate of $\mathrm{Kv} 4$ protein, and slows the inactivation kinetics and speeds the rate of recovery from inactivation of $\mathrm{Kv} 4$ channels (An et al., 2000; Shibata et al., 2003; Jerng et al., $2004 \mathrm{~b})$. The pattern of association and colocalization of Kv4.2, Kv4.3, and KChIP1KChIP4 varies across brain regions and cell types (Rhodes et al., 2004; Strassle et al., 2005), suggesting specific yet undefined requirements for precise compositions of Kv4 $\alpha$ subunits and KChIPs in channel complexes.

Here we show that genetic ablation of Kv4.2 does not lead to changes in expression or localization of Kv4.3 in any neurons examined, regardless of the extent of Kv4.2 and Kv4.3 coexpression found in WT neurons. The different subfields of hippocampus provide a striking example of this lack of compensatory change in Kv4.3. Hippocampal neurons are divided into three groups based on their cellular and subcellular expression of Kv4.2 and Kv4.3 $\alpha$ subunits: neurons that express Kv4.2 but not Kv4.3 (e.g., CA1 pyramidal neurons), neurons that coexpress colocalized Kv4.2 and Kv4.3 (e.g., dentate granule and CA3 pyramidal cells), and neurons that express Kv4.3 but not Kv4.2 (interneurons throughout all hippocampal subfields). Increased expression of Kv4.3 in neurons that normally express Kv4.2 and Kv4.3 (e.g., hippocampal dentate granule and CA3 pyramidal cells) and/or ectopic expression of Kv4.3 in neurons that normally express only Kv4.2 (e.g., hippocampal CA1 pyramidal cells) might be anticipated given the prominent role proposed for Kv4-containing somatodendritic A-type channels and the surprisingly normal behavior of $\mathrm{Kv} 4.2^{-1-}$ mice. Moreover, the high-level expression of Kv4.2 in many brain neurons, the coexpression of Kv4.2 and Kv4.3 in a subset of these cells, and the overall similarity in the functional properties of Kv4.2 and Kv4.3 homomeric and heteromeric channels in heterologous systems imply that upregulation or ectopic expression of Kv4.3 is a parsi-
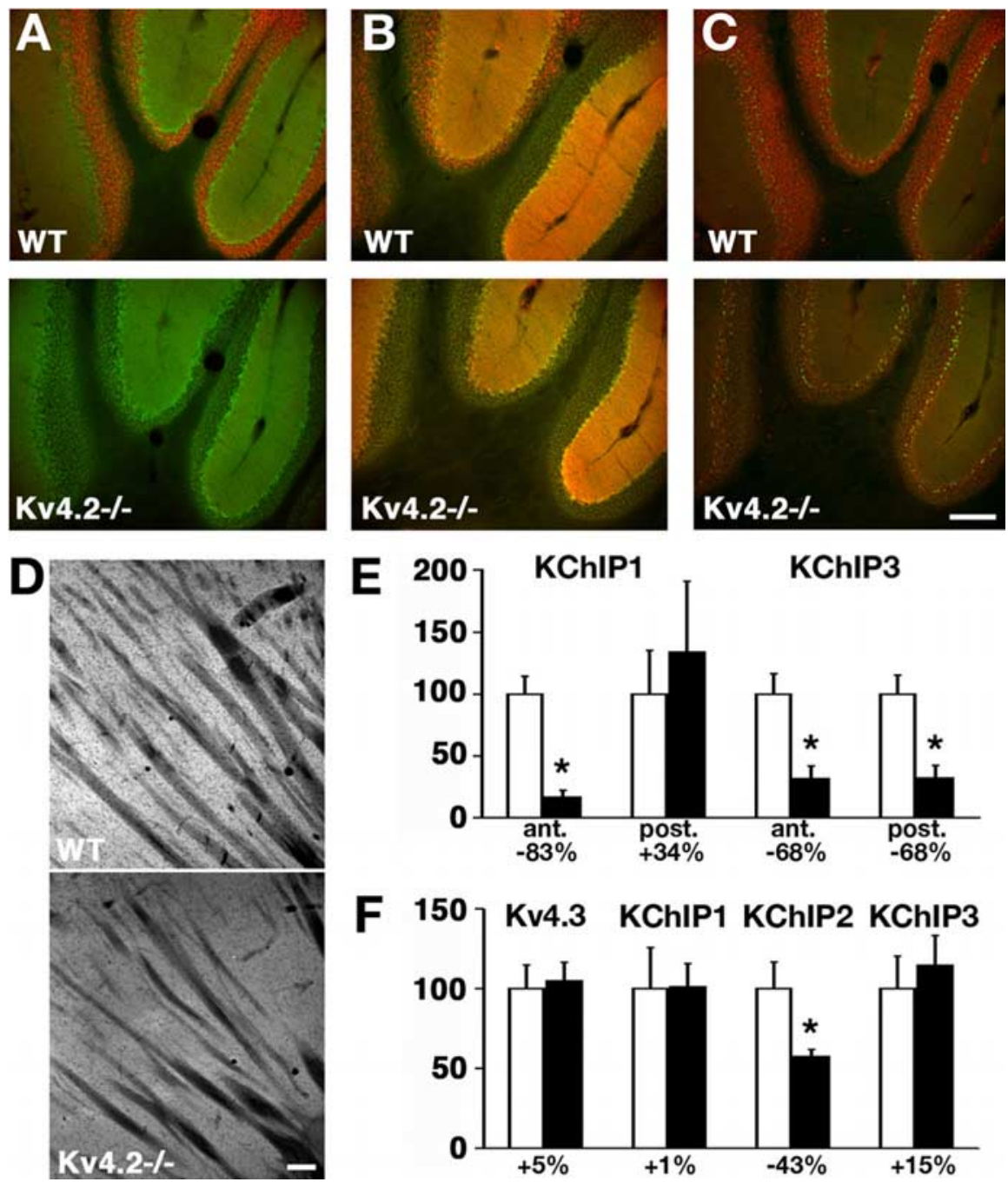

Figure 3. Expression of Kv4 and KChIP subunits in WT and $K v 4.2^{-/-}$cerebella and striata. $\boldsymbol{A}-\boldsymbol{C}$, Immunostaining of WT and Kv4.2 $2^{-1-}$ sagittal cerebellar sections for Kv4.2 (A), KChIP1 (B), and KChIP3 $(\boldsymbol{C})$ in red and Slo1 $(\boldsymbol{A}, \boldsymbol{B})$ and Kv1.2 $(\boldsymbol{C})$ in green. Anterior, Left; posterior, right. Scale bar, $200 \mu \mathrm{m}$. D, Staining of KChIP2 in WT and Kv4.2- $2^{--}$striatum. Scale bar, $100 \mu \mathrm{m} . \boldsymbol{E}$, , was set at $100 \%$, and the remaining three mean values were subsequently normalized. Values are mean \pm SE.

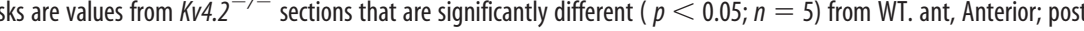
posterior. $\boldsymbol{F}$, Fluorescence intensity measurements from $400 \times 400 \mu \mathrm{m}$ square striatal fields from five WT (white bars) and four $K v 4.2^{-1-}$ (black bars) mice, as described in $\boldsymbol{D}$.

monious compensatory mechanism for loss of neuronal Kv4.2. It should be noted that, because of lack of a specific antibody, we were unable to assay expression of the other Kv4 family member Kv4.1, which, although expressed at very low levels in adult rat brain (Serodio and Rudy, 1998), does not preclude a compensatory role in $K v 4.2^{-/-}$.

That we did not observe compensatory changes in Kv4.3 expression in $K v 4.2^{-/-}$hippocampus is consistent with recent studies showing a lack of changes in Kv4.3 expression between WT and $K v 4.2^{-1-}$ on immunoblots of hippocampal membrane fractions (Chen et al., 2006). Moreover, these studies showed a specific and complete loss of dendritic A-type Kv currents from apical dendrites of CA1 pyramidal neurons (Chen et al., 2006), consistent with our staining of CA1 stratum radiatum that revealed a loss of Kv4.2 and no upregulation of Kv4.3 (Figs. 1, 2). This lack of Kv4.3 upregulation is also consistent with recent 
studies showing lack of Kv4.3 upregulation in ventricular myocytes (Guo et al., 2005) and dorsal spinal cord nociceptive neurons (Hu et al., 2006) in these Kv4.2 $2^{-/-}$mice. The complete loss of $I_{\mathrm{to}, \mathrm{f}}$ in $\mathrm{Kv} 4.2^{-1-}$ ventricle suggests that Kv4.2 is an obligatory subunit of $\mathrm{Kv} 4.2 / \mathrm{Kv} 4.3$ heteromeric channels that underlie mouse ventricular $I_{\text {to,f }}$. However, heterologous cells (Guo et al., 2005) and hippocampal and neocortical interneurons (Rhodes et al., 2004) all express functional Kv4.3 homotetrameric channels in the absence of $\mathrm{Kv} 4.2$. Why a simple upregulation of $\mathrm{Kv} 4.3$ does not occur in $K v 4.2^{-1-}$ brain is unclear but presumably reflects subtleties in regulatory mechanisms or other aspects of neuronal function that are not yet well understood.

Given the lack of compensatory changes in Kv4.3, it was surprising to find such a pronounced downregulation of KChIP expression in $K v 4.2^{-1-}$ brains. Depending on the brain region examined, deletion of Kv4.2 induced a strong reduction in expression of KChIP1, KChIP2, or KChIP3. Although all three subunits were expressed in these regions, in hippocampus, the lack of Kv4.2 induced a decreased expression of KChIP2 and KChIP3, in striatum KChIP2, and in cerebellum KChIP1 and KChIP3. The extent of reduction in levels of KChIP expression we observed in different regions correlates well with the extent of their colocalization (and presumably association) with Kv4.2 in WT (and rat) brain.

The different subfields of hippocampus provide a conspicuous example of this. Ablation of Kv4.2 expression in CA1 pyramidal neurons generates a profound $(\sim 80 \%)$ decrease in expression of KChIP2 (the major KChIP isoform in these neurons). In $K v 4.2^{-/-}$hippocampus, dentate granule and CA3 pyramidal neurons exhibit less pronounced reductions in KChIP2 and KChIP3 expression, whereas expression of KChIP1 (expressed mainly in interneurons) remains mostly unaffected. These results are consistent with expression levels of KChIPs in neurons generally tracking overall levels of Kv4 expression. However, there exists another level of regulation, such that depending on neuronal type, expression of a single KChIP is more greatly affected by loss of Kv4.2 than are other KChIPs expressed in those cells. A prominent example of this is dentate granule cells, which normally express Kv4.2, Kv4.3, KChIP2, and KChIP3. The loss of Kv4.2 in these cells has a more profound effect on KChIP3 expression level than that of KChIP2. The cell-specific changes in KChIP expression presumably reflect the requirement for specific Kv4:KChIP combinations in different neurons, as proposed previously (Rhodes et al., 2004). Interestingly, KChIP2, the only cardiac KChIP, is virtually eliminated in ventricles of $K v 4.2^{-1-}$ hearts, although Kv4.3 is still expressed at high levels (Guo et al., 2005).

These results together suggest that powerful yet complex compensatory mechanisms exist to govern expression of A-type potassium channel subunits in neurons. Our results raise a number of mechanistic questions. First, how do cells that normally express both $\mathrm{Kv} 4.2$ and $\mathrm{Kv} 4.3$ maintain remarkably normal levels of Kv4.3 expression in complete absence of Kv4.2? Cells such as CA3 pyramidal neurons can apparently sense and maintain expression levels of Kv4.3, whereas levels of overall $\mathrm{Kv} 4$ expression and presumably A-type currents decrease. Invertebrate neurons have a mechanism for sensing Kv4 subunit expression level independent of their functional state (MacLean et al., 2003). Our data suggest that mammalian neurons may have an analogous system.

Second, how are expression levels of KChIPs so strongly tied to Kv4 and/or Kv4.2 expression levels? Our results suggest that, in $K v 4.2^{-1-}$ brain, KChIP expression is virtually eliminated in neu- rons that normally express Kv4.2 alone, decreased (halved?) in neurons that coexpress Kv4.2 and Kv4.3, and unaffected in neurons that express Kv4.3 alone. This suggests that expression levels of Kv4 and KChIPs are tightly coupled and provide compelling evidence that the predominant if not exclusive role for neuronal $\mathrm{KChIPs}$ is as auxiliary subunits of $\mathrm{Kv} 4$ channels. It also suggests a feedback mechanism to ensure that free KChIPs do not accumulate. This could be achieved by coupling KChIP gene expression to overall Kv4 expression levels and/or through posttranscriptional/posttranslational mechanisms. Previously, we found that KChIP coexpression led to increased steady-state expression levels of Kv4.2 by increasing Kv4.2 protein stability (Shibata et al., 2003); loss of reciprocal stabilization of KChIPs that could occur during association with $\mathrm{Kv} 4$ subunits could result in lower KChIP expression levels in $\mathrm{Kv} 4.2^{-/-}$.

Last, how do different neurons selectively regulate individual KChIPs? Dentate granule cells, which express Kv4.2, Kv4.3, KChIP2, and KChIP3, respond to loss of Kv4.2 by dramatically decreasing levels of KChIP3 and to a lesser extent KChIP2. However, in striatal neurons, which also express these same four subunits, KChIP2 levels are decreased but KChIP3 levels are not. Complex, subtype-specific regulatory mechanisms, which can selectively target the expression of one KChIP over another in a single neuron, presumably exist to ensure expression and incorporation of specific KChIPs into Kv4-containing A-type channel complexes.

\section{References}

An WF, Bowlby MR, Betty M, Cao J, Ling HP, Mendoza G, Hinson JW, Mattsson KI, Strassle BW, Trimmer JS, Rhodes KJ (2000) Modulation of A-type potassium channels by a family of calcium sensors. Nature 403:553-556.

Bekele-Arcuri Z, Matos MF, Manganas L, Strassle BW, Monaghan MM, Rhodes KJ, Trimmer JS (1996) Generation and characterization of subtype-specific monoclonal antibodies to $\mathrm{K}^{+}$channel alpha- and betasubunit polypeptides. Neuropharmacology 35:851-865.

Birnbaum SG, Varga AW, Yuan LL, Anderson AE, Sweatt JD, Schrader LA (2004) Structure and function of Kv4-family transient potassium channels. Physiol Rev 84:803-833.

Chen X, Yuan L-L, Zhao C, Birnbaum SG, Frick A, Jung WE, Schwarz TL, Sweatt JD, Johnston D (2006) Deletion of Kv4.2 gene eliminates dendritic A-type $\mathrm{K}^{+}$current and enhances induction of long-term potentiation in hippocampal CA1 pyramidal neurons. J Neurosci 26:12143-12151.

Guo W, Jung WE, Marionneau C, Aimond F, Xu H, Yamada KA, Schwarz TL, Demolombe S, Nerbonne JM (2005) Targeted deletion of Kv4.2 eliminates $I(t o, f)$ and results in electrical and molecular remodeling, with no evidence of ventricular hypertrophy or myocardial dysfunction. Circ Res 97:1342-1350.

Hu HJ, Carrasquillo Y, Karim F, Jung WE, Nerbonne JM, Schwarz TL, Gereau RW (2006) The Kv4.2 potassium channel subunit is required for pain plasticity. Neuron 50:89-100.

Jerng HH, Qian Y, Pfaffinger PJ (2004a) Modulation of Kv4.2 channel expression and gating by dipeptidyl peptidase 10 (DPP10). Biophys J $87: 2380-2396$

Jerng HH, Pfaffinger PJ, Covarrubias M (2004b) Molecular physiology and modulation of somatodendritic A-type potassium channels. Mol Cell Neurosci 27:343-369.

MacLean JN, Zhang Y, Johnson BR, Harris-Warrick RM (2003) Activityindependent homeostasis in rhythmically active neurons. Neuron 37:109-120.

Misonou H, Menegola M, Buchwalder L, Park EW, Meredith A, Rhodes KJ, Aldrich RW, and, Trimmer JS (2006) Immunolocalization of the $\mathrm{Ca}^{2+}$. activated $\mathrm{K}^{+}$channel Slol in axons and nerve terminals of mammalian brain and cultured neurons. J Comp Neurol 496:289-302.

Nadal MS, Ozaita A, Amarillo Y, Vega-Saenz de Miera E, Ma Y, Mo W, Goldberg EM, Misumi Y, Ikehara Y, Neubert TA, Rudy B (2003) The 
CD26-related dipeptidyl aminopeptidase-like protein DPPX is a critical component of neuronal A-type $\mathrm{K}^{+}$channels. Neuron 37:449-461.

Rhodes KJ, Strassle BW, Monaghan MM, Bekele-Arcuri Z, Matos MF, Trimmer JS (1997) Association and colocalization of the $\operatorname{Kv} \beta 1$ and $\operatorname{Kv} \beta 2$ $\beta$-subunits with $\mathrm{Kvl} \alpha$-subunits in mammalian brain $\mathrm{K}^{+}$channel complexes. J Neurosci 17:8246-8258.

Rhodes KJ, Carroll KI, Sung MA, Doliveira LC, Monaghan MM, Burke SL, Strassle BW, Buchwalder L, Menegola M, Cao J, An WF, Trimmer JS (2004) KChIPs and Kv4 $\alpha$ subunits as integral components of A-type potassium channels in mammalian brain. J Neurosci 24:7903-7915.

Serodio P, Rudy B (1998) Differential expression of Kv4 K ${ }^{+}$channel subunits mediating subthreshold transient $\mathrm{K}^{+}$(A-type) currents in rat brain. J Neurophysiol 79:1081-1091.
Shibata R, Misonou H, Campomanes CR, Anderson AE, Schrader LA, Doliveira LC, Carroll KI, Sweatt JD, Rhodes KJ, Trimmer JS (2003) A fundamental role for KChIPs in determining the molecular properties and trafficking of Kv4.2 potassium channels. J Biol Chem 278:36445-36454.

Strassle BW, Menegola M, Rhodes KJ, Trimmer JS (2005) Light and electron microscopic analysis of KChIP and Kv4 localization in rat cerebellar granule cells. J Comp Neurol 484:144-155.

Trimmer JS (1991) Immunological identification and characterization of a delayed rectifier $\mathrm{K}^{+}$channel polypeptide in rat brain. Proc Natl Acad Sci USA 88:10764-10768.

Zagha E, Ozaita A, Chang SY, Nadal MS, Lin U, Saganich MJ, McCormack T, Akinsanya KO, Qi SY, Rudy B (2005) DPP10 modulates Kv4-mediated A-type potassium channels. J Biol Chem 280:18853-18861. 